\title{
Successful disinfection of femoral head bone graft using high hydrostatic pressure
}

\author{
Michiel A. J. van de Sande · Judith V. M. G. Bovée • Mark van Domselaar • \\ Marja J. van Wijk • Ingrid Sanders • Ed Kuijper
}

Received: 2 October 2017/ Accepted: 12 December 2017/Published online: 20 December 2017

(C) The Author(s) 2017. This article is an open access publication

\begin{abstract}
The current standard for sterilization of potentially infected bone graft by gamma irradiation and thermal or chemical inactivation potentially deteriorates the biomechanical properties of the graft. We performed an in vitro experiment to evaluate the use of high hydrostatic pressure (HHP); which is widely used as a disinfection process in the food processing industry, to sterilize bone grafts. Four femoral heads were divided into five parts each, of which 16 were contaminated (in duplicate) with $10^{5}-$
\end{abstract}

Michiel A. J. van de Sande, Judith V. M. G. Bovée, Mark van Domselaar, Marja J. van Wijk, Ingrid Sanders and Ed Kuijper have contributed equally to this work.

M. A. J. van de Sande ( $\square)$

Department of Orthopaedic Surgery, Leiden University Medical Center, Leiden, Zuid-Holland, The Netherlands e-mail: majvandesande@lumc.nl

J. V. M. G. Bovée

Department of Pathology, Leiden University Medical

Center, Leiden, Zuid-Holland, The Netherlands

M. van Domselaar

Research Department, TDI-BV, Wageningen, Gelderland, The Netherlands

M. J. van Wijk

Medical Department, BISLIFE Foundation, Leiden,

Zuid-Holland, The Netherlands

I. Sanders · E. Kuijper

Department of Medical Microbiology, Leiden University

Medical Center, Leiden, Zuid-Holland, The Netherlands
$10^{7} \mathrm{CFU} / \mathrm{ml}$ of Staphylococcus epidermidis, Bacillus cereus, or Pseudomonas aeruginosa or Candida albicans, respectively. Of each duplicate, one sample was untreated and stored similarly as the treated sample. The remaining four parts were included as sterile control and non-infected control. The 16 parts underwent HHP at the high-pressure value of $600 \mathrm{MPa}$. After HHP, serial dilutions were made and cultured on selective media and into enrichment media to recover low amounts of microorganism and spores. Three additional complete femoral heads were treated with 0,300 and $600 \mathrm{MPa} \mathrm{HHP}$ respectively for histological evaluation. None of the negative-control bone fragments contained microorganisms. The measured colony counts in the positive-control samples correlated excellent with the expected colony count. None of the HHP treated bone fragments grew on culture plates or enrichment media. Histological examination of three untreated femoral heads showed that the bone structure remained unchanged after HHP. Sterilizing bone grafts by high hydrostatic pressure was successful and is a promising technique with the possible advantage of retaining biomechanical properties of bone tissue.

Keywords Disinfection - Bone graft · Hydrostatic pressure $\cdot$ Microorganisms

\section{Abbreviations \\ CFU Colony forming units}


$\mathrm{McF} \quad$ MacFarland

HPP High pressure processing

BHI Brain heart infusion

PBS Phosphate-buffered saline

SAB Sabouraud medium

SBA $5 \%$ sheep blood agar medium

\section{Introduction}

Sterilization of bone and soft tissue allograft at present is mainly performed using extracorporeal irradiation or autoclaving, generally followed by freeze-dried preservation in a bone bank (Diehl et al. 2005). However, sterilization by gamma irradiation and thermal or chemical inactivation of allografts and other biomaterials, considered for tissue regeneration and reconstruction, is associated with deterioration of the mechanical, physical and biological properties of the bony implant (Barth et al. 2011; Kaminski et al. 2012).

High hydrostatic pressure (HHP), successfully used in the food processing industry to disinfect and prolong expiration dates, has been proposed as a new entity for bone graft disinfection and preservation. In addition, HHP could be an alternative for irradiation and reimplantation of resected bone, after bone tumor resection (Diehl et al. 2003; Weber et al. 2008; Naal et al. 2005). The hypothesized additional advantage of HHP in the sterilization of bone graft would be the potential to retain biomechanical properties of the graft (Diehl et al. 2006; Naal et al. 2008). In 2007 Diehl et al. were the first to demonstrate that HHP can effectively devitalize and sterilize bone grafts, cartilage and tendon in vitro while leaving the tissues' mechanical properties unimpaired, thus allowing for reimplantation of the resected tissue (Diehl et al. 2007). In addition, the bacteriostatic characteristics of HHP in contaminated and infected bone and implants at 300-600 MPa were reported, although no complete sterilisation of infected bone material could be achieved (Gollwitzer et al. 2009). To our knowledge, no other study groups have published on the use of HHP in bone graft sterilization. To explore further possible use in biomedical practice, we were interested in the application of HHP technology to sterilize or disinfect bone grafts. The first step allowing reimplantation of bone graft is to assess the sterilization process of bone grafts using the HHP process. Hereafter long-term preservation, structural integrity and biomechanical properties of the bone graft could be thoroughly assessed in further studies.

Our aim was to explore the sterilising properties of HHP treatment for bone graft in an in vitro experiment. We hypothesize that HHP treatment of contaminated bone graft results in a minimal $\log 4-5$ reduction of the amount of induced bacterial contamination. We chose four commonly cultured microorganisms representative for different groups of microorganisms, found as contaminants of bone grafts used in the Netherlands (Bonebank, BISLIFE, Leiden, The Netherlands).

\section{Materials and methods}

Seven fresh frozen femoral heads, that were unsuitable for transplantation for reasons other than infectious disease (Bonebank BISLIFE, Leiden The Netherlands), were selected. Donors and femoral heads were cultured by standard procedures (van de Pol et al. 2007) (swab culture of the surface of the whole femoral head) and all were found negative. Using an oscillation saw in the surgical theatre under down flow and sterile circumstances, four femoral heads were divided into 5 equal parts each (Fig. 1). They were again packaged using a sterile container and transported to the department for clinical microbiology, in a cooled box. Sixteen parts were contaminated in a sterile environment and subsequently sealed in a plastic bag containing $100 \mathrm{ml}$ of phosphate-buffered saline (PBS) with $10^{5-7} \mathrm{CFU} / \mathrm{ml}$ of Staphylococcus epidermidis (ATCC 14990), Bacillus cereus (ATCC 14579), Pseudomonas aeruginosa (ATCC 27853) and Candida albicans (ATCC 10231), in duplicate, respectively (Fig. 1). As controls, four parts were not contaminated but directly packaged in $100 \mathrm{ml}$ of the same phosphate-buffered saline (PBS); $\mathrm{pH} 7.5$, in a sealed sterile plastic bag.

Bacterial infection, dilution series and cultures

To obtain a starting concentration of $10^{5} \mathrm{CFU} / \mathrm{ml}$ of $S$. epidermidis and $P$. aeruginosa, $1 \mathrm{ml}$ of a concentration of $10^{7} \mathrm{CFU} / \mathrm{ml}$ was diluted in $99 \mathrm{ml}$ Phosphate buffered salt (PBS). The concentration of $10^{7} \mathrm{CFU} / \mathrm{ml}$ was estimated by McFarland and confirmed by 


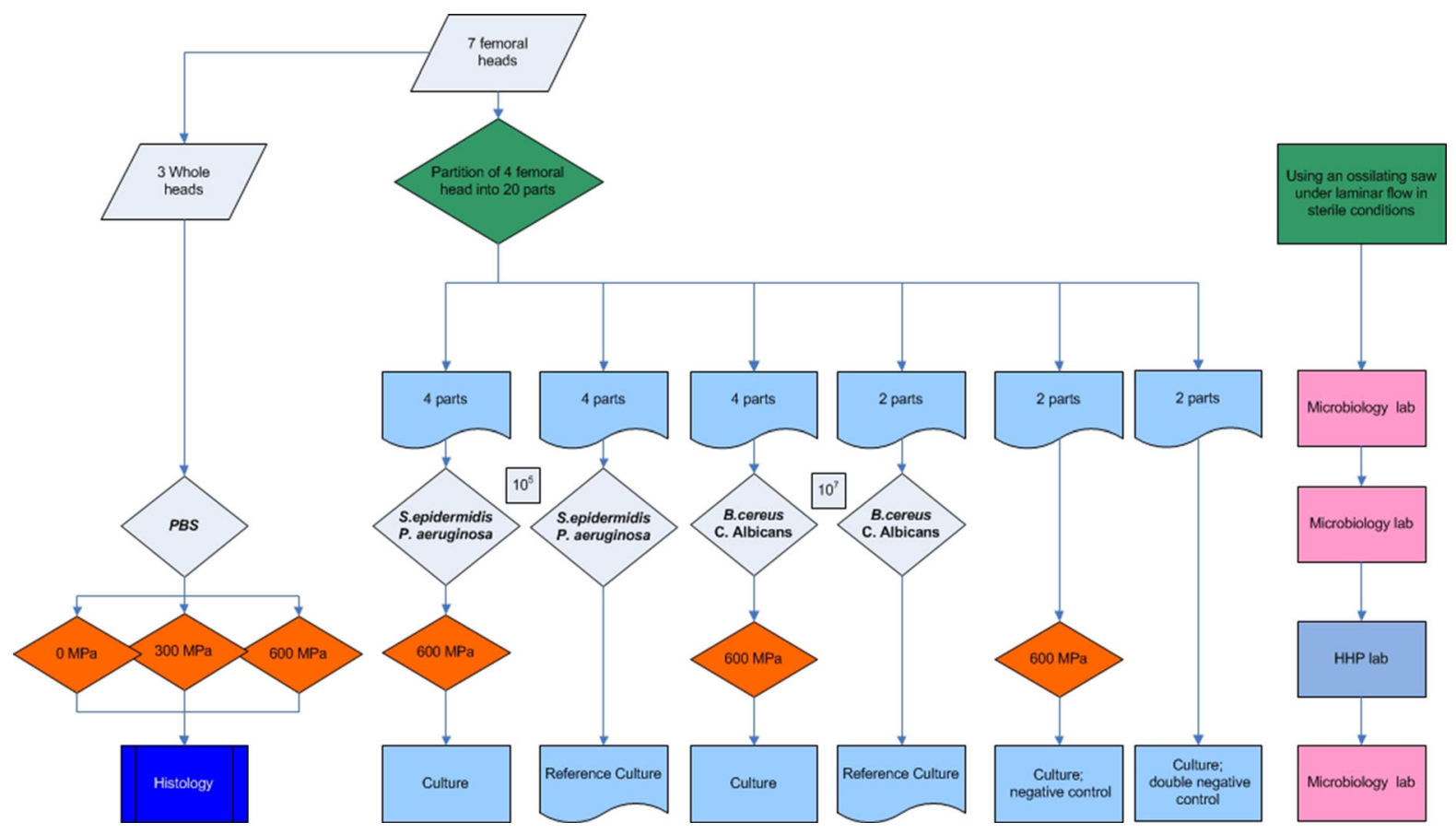

Fig. 1 Flowchart for materials and methods

culturing serial dilutions onto 5\% sheep blood agar plates (SBA; bioMérieux, Marcy l'Etoile, France). Each contaminated femoral head was contaminated with a total volume of $100 \mathrm{ml}$.

For C. albicans and B. cereus, each sample was contaminated with $10^{7} \mathrm{CFU} / \mathrm{ml}$. Similar as described above; a starting concentration was made using a McFarland dilution and confirmed by control cultures.

All contaminated bags and controls were labelled, vacuum-sealed in sterile bags and preserved and transported at cool conditions $\left( \pm 4{ }^{\circ} \mathrm{C}\right)$ to undergo HHP at a secondary location, at Tournois Dynamic Innovations (TDI BV) Helmond, The Netherlands. The mechanism and use of HHP to disinfect bone and soft tissue graft was earlier described Diehl et al. (2007). Its mechanism relies on the cell disruptive properties of HPP while not affecting the extra cellular matrix or bone scaffold. To ensure the sterile containment and packaging, $150 \times 200 \mathrm{~mm} 170$ micron, plastic bags (Hevel Super Export Plus) were used. The plastic bags were sterilized by gamma irradiation. Pascalisation followed at TDI the next day, for $30 \mathrm{~min}$ at $600 \mathrm{MPa}$ or for $30 \mathrm{~min}$ at $300 \mathrm{MPa}$ in a Hiperbaric 55 (one whole femoral head only). Temperature of the process water before the pressure increase was
14-15 ${ }^{\circ} \mathrm{C}$ and the samples went in at $7{ }^{\circ} \mathrm{C}$. The Adiabatic temperature rise caused by the increased pressure within the vessel was calculated using the following formula; for pure organic substances (Cp $\AA$ $2 \mathrm{~kJ} / \mathrm{kg} \mathrm{K}$ ) the adiabatic temperature rise is often approximated by $\mathrm{DTad}=\mathrm{DHr} / 2=3{ }^{\circ} \mathrm{C} / 100 \mathrm{MPa}$ increase in pressure. Maximum temperature therefore never exceeded over $33{ }^{\circ} \mathrm{C}$ at $600 \mathrm{MPa}$.

After HHP, the samples were returned to the microbiology department in the LUMC under cooled circumstances at $4{ }^{\circ} \mathrm{C}$ within $24 \mathrm{~h}$ after HHP. All contaminated bags and controls were opened and cultured in a sterile environment. One $\mathrm{ml}$ of suspension was diluted in PBS $\left(10^{6}-10^{5}-10^{4}-10^{3}-10^{2}\right)$ and $50 \mu \mathrm{l}$ of the mixture was cultured on SBA plates. From the undiluted samples $200 \mu \mathrm{l}$ was plated out. All plates were incubated for $48 \mathrm{~h}$ at $37^{\circ} \mathrm{C}$. Additionally, the femoral head fragment was inoculated in an enrichment medium consisting of $100 \mathrm{ml}$ liquid Brain Hart Infusion (BHI) broth. After $48 \mathrm{~h}$ incubation at $37{ }^{\circ} \mathrm{C}$, BHI was sub-cultured to SBA, cysteine lactose electrolyte deficient agar (CLED; bioMérieux, Marcy l'Etoile, France) and Sabouraud agar (SAB; bioMérieux, Marcy l'Etoile, France) to examine growth of bacteria and yeasts within the bone graft. 
Histological evaluation

Three additional intact femoral heads were not contaminated but underwent the same protocol as the contaminated samples. The three femoral heads were packaged into a sterile environment into a plastic bag filled with $100 \mathrm{ml}$ PBS and vacuum-sealed. One was left untreated, the other two underwent either HHP with 300 or $600 \mathrm{MPa}$ and all were stored for 5 days at cool temperature (around $4{ }^{\circ} \mathrm{C}$ ). The femoral heads were then cut in half and the central part of the femoral head was fixed in formalin, decalcified using formic acid, and embedded in paraffin. Four $\mu \mathrm{m}$ sections were stained with haematoxylin and eosin. All were standard procedures.

\section{Results and discussion}

Microbiology

Both S. epidermidis and P. aeruginosa could not be cultured after $\mathrm{HHP}$ at $600 \mathrm{MPa}$. Starting with a concentration of $10^{5}$ this resulted in a $\log 5$ reduction. Similarly, we found a $\log 7$ reduction for $B$. cereus and C. albicans (Fig. 2a, b). The control of the starting concentration confirmed that the administered concentration was correct. The control bags that did not undergo HHP treatment, showed bacterial growth as expected (Fig. 2a, b). The concentration after transport to and from the HHP facility was somewhat lower than the starting concentration. Sample no. 9 was contaminated with a gram-positive rod, not belonging to the genus Bacillus which was therefore considered contamination during experimental protocol.

\section{Histology}

Haematoxylin and eosin staining of the three intact femoral heads revealed that microscopically the bone structure remained intact after HHP treatment (Fig. 3). There seemed to be a decrease in nuclear staining of the osteocytes after HHP treatment; in the untreated bone vital osteocytes were easily identified, after $300 \mathrm{MPa}$ vital osteocytes were still present but in the treated bone with $600 \mathrm{MPa}$ the lacunae appeared empty.

Contamination of bone tissue harvested in living donors or in a post-mortem explantation setting is estimated at $10-30 \%$ of all grafts, predominantly with $S$ epidermidis (Schubert et al. 2012; Journeaux et al. 1998; Mathijssen et al. 2013; Sommerville et al. 2000). Although these high contamination rates underline the importance of reliable bone graft disinfection after harvesting, the risk for disease transmission through bone graft implantation remains extremely low (van de Pol et al. 2007; Sommerville et al. 2000; Tomford et al. 1990; Chiu et al. 2004). Graft disinfection with use of antibiotic solution is considered as insufficient (Deijkers et al. 1996). Therefore radiation treatment is commonly advised to control exogenous contamination during retrieval procedure and graft processing (Deijkers et al. 1996). However, radiation is reported to be deleterious to the biomechanical properties of bone graft (Mitchell et al. 2004). This negative influence of radiation on the structural integrity and biomechanical properties of bone grafts may be reduced by decreasing the radiation dosage to $15 \mathrm{kGy}$, but new reliable disinfection protocols providing optimal biomechanical properties of bone graft seem appropriate (Nguyen et al. 2013).

High hydrostatic pressure (HHP) at present is widely used in the food industry as it offers multiple opportunities for pasteurization, enzyme inactivation and freezing of foods utilizing the HHP induced pressure shift (San Martín et al. 2002). By changing the absolute pressure, hold time of the pressure, temperature and or chemical environment, the induced molecular changes can be either permanent or reversible. In general, a pressure-transferring medium, usually water, is necessary to provide uniform or isotropic transmission of pressure throughout a mass independent of size, shape, and composition. At higher pressures (300-600 MPa) most microorganisms and spores are reported to be (partially) eliminated (Knorr 1999). HHP even inactivates mycobacteria, spores of Bacillus species and certain enveloped and nonenveloped viruses (de Souza et al. 2013; Olivier et al. 2011; Kishida et al. 2013). The anti-microbial effect of HHP is well understood in food preservation but clinical use remains limited.

We found that HHP resulted in a significant reduction of the amount of bacteria and yeasts in contaminated femoral heads, which would make HHP suitable for disinfection of bone graft tissues to be used in (orthopaedic) surgery. We demonstrated that HHP reduced the amount of bacteria ( $S$. epidermidis, $P$. 
Fig. 2 Test and retest (a, b) showing no growth for bacteria ( $S$. epidermidis, $P$. aeruginosa and B. cereus) and yeast (C. albicans) after $600 \mathrm{MPa}$ treatment. 1. Real starting concentration (CFU/ml in hundreds), 2. Concentration after transport without HHP (CFU/ml in hundreds), 3. Concentration after transport and $600 \mathrm{MPa} \mathrm{HHP}$ (CFU/ml in hundreds)
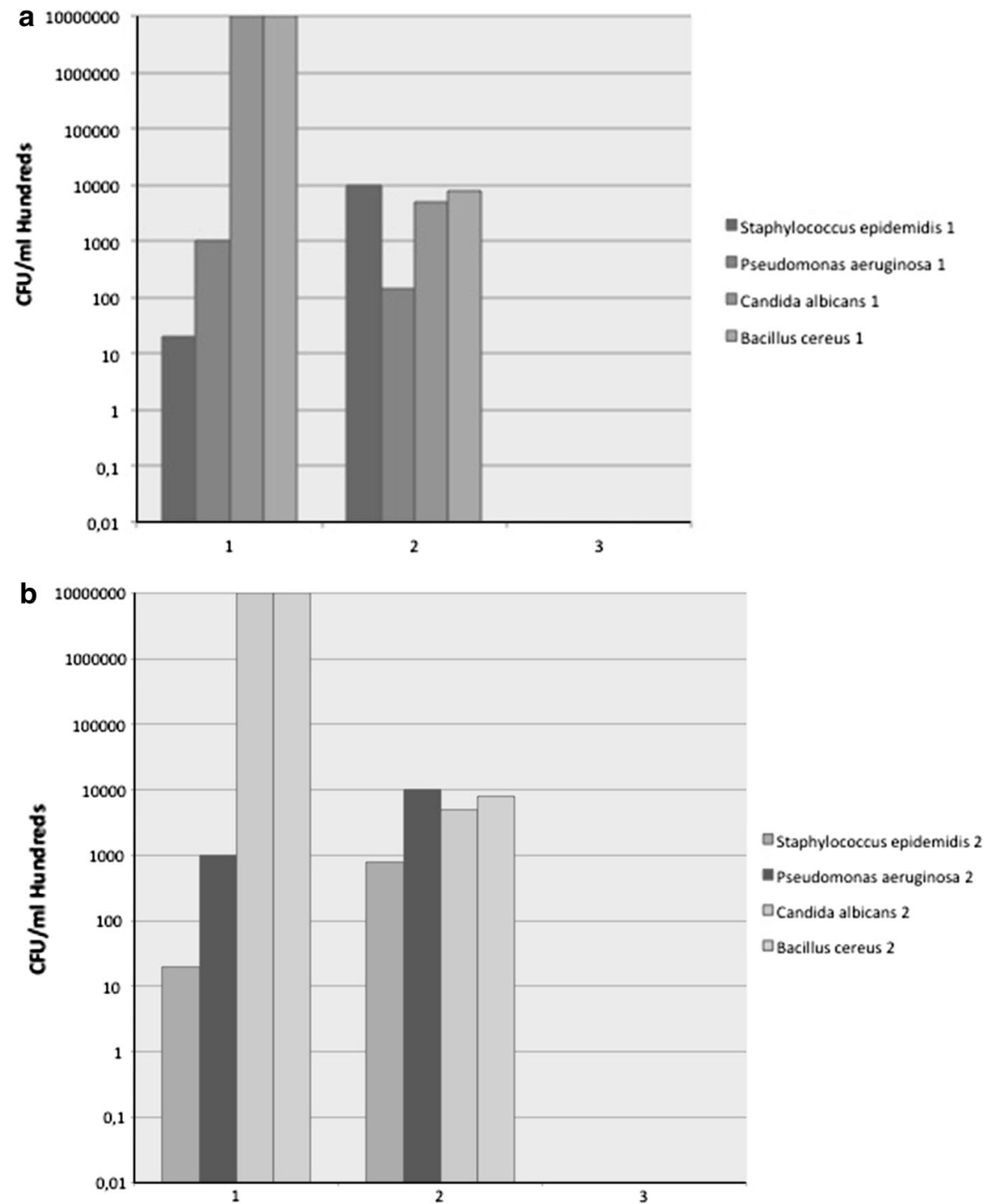

aeruginosa and $B$. cereus) and yeast (C. albicans) by at least $\log 5$. These results are comparable to the disinfection properties of radiation treatment of bone grafts with 15-25 kGy (Nguyen et al. 2008, 2013). Though we achieved complete sterilization and fulfil to the requirements of the European Pharmacopoeia, we still consider the experiments as a pilot study. The next step is to enlarge our study with inclusion of other types of grafts and additional but different microorganisms.

Our results are in contrast to the findings of Gollwitzer et al. (2009), who reported significant failure rates after HHP disinfection of contaminated bone. Although Gollwitzer found a clear effect using
$300 \mathrm{MPa}$ on $S$. aureus contaminated stainless steel screws, no significant effect was found on artificially infected bone grafts harvested from patients with aseptic osteoarthritis. These findings are in contrast with our results, which may be explained by a baroprotective effect of the surrounding inflamed bone in some of the samples tested by Gollwitzer. This hypothesis is supported by their observation that various bone specimens could be completely disinfected, whereas others proved resistant to treatment with unaffected bacterial growth (Gollwitzer et al. 2009). Additionally, they underlined the possibility of barosensitive and barotolerant microorganisms and strains, which was also postulated by Alpas et al. 


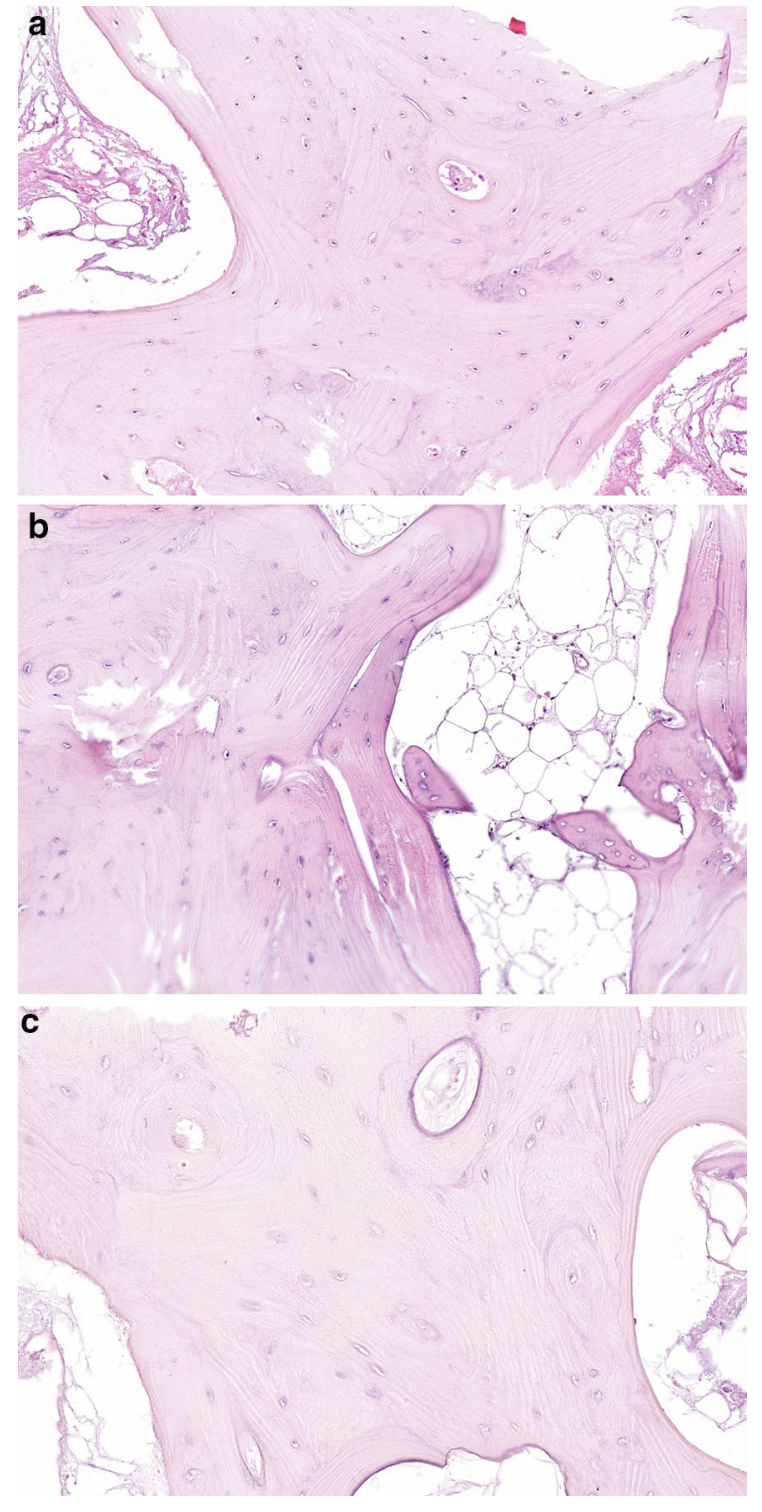

Fig. 3 a Femoral head number 1 fully processed and conserved but without HHP treatment, H\&E staining shows intact structure of the bone and vital osteocytes, $\mathbf{b}$ femoral head number 2 after $300 \mathrm{MPa}$ HHP, H\&E staining shows intact structure of the bone with some vital osteocytes, c femoral head number 3 after $600 \mathrm{MPa} H H \mathrm{P}, \mathrm{H} \& \mathrm{E}$ staining shows intact structure of the bone with lack of nuclear staining of osteocytes

(1999). Further evaluation of susceptibility of different microorganisms, strains and spores is therefore warranted.

We also demonstrated that the bone structure remains microscopically unaltered after HHP. Additional micro-CT evaluation after HHP treatment in the future will possibly allow for more detail on the structural integrity of bone grafts after HHP. In addition, we report that viable osteocytes seem to decrease in numbers with increased pressure of HHP, which was also reported previously by others (Naal et al. 2008; Diehl et al. 2008). However, the number of femoral heads in our study is too low to draw definitive conclusions. Also, we cannot rule out that the clinical history of these selected femoral heads may have included osteoarthritis, osteonecrosis or fracture. These three underlying diseases may have influenced the initial viability of the osteocytes and may have altered bone architecture.

The ultimate balance between the high pressure necessary to disinfect the bone graft and maximum pressure tolerated by the osteocytes needs further attention. Recent publications have underlined the important role of osteocyte apoptosis in bone remodelling through RANKL expression and sclerostin secretion, inducing osteoclastogenesis and osteoclastic bone resorption (Graham et al. 2013; Pajevic et al. 2010).

The osteoconductive and osteoinductive properties of HHP treated bone graft were indirectly explored earlier, using HHP treated bone as scaffold for seeded viable osteoblast-like cells prepared from donor bones (Schauwecker et al. 2011). Schauwecker et al. reported that independent of the applied HHP protocol $74 \%$ of the seeded cells adhered to the bone matrix. They anticipated that HHP treatment up to $600 \mathrm{MPa}$ causes no alterations in bone matrix that could impair the osteoconductivity of the graft. Their conclusions again warrant further exploration of the osteoconductive, but also osteoinductive potential of HHP treated bone tissues.

One of the limitations of our study is that only four different microorganisms were used. Additional tests for less frequent contaminants are proposed. The ultimate effect on the survival of spores of the Bacillus was not fully investigated as we only cultured up to $92 \mathrm{~h}$. Moreover, our study only focuses on contaminations occurring before storage of the bone grafts. More information is warranted on possible late contamination of the bone graft during transport and storage just before use in the surgical theatre. Additionally, as this disinfection technique possibly allows storage of the bone graft in temperatures above zero degrees, direct use during surgery would be possible, but the effect of cooled preservation of bone graft on 
the bacterial disinfection and the vitality of the allografts osteocytes need further evaluation. In addition, the final concentration of bacteria and yeasts after transport were somewhat lower than the starting concentration. This can be explained by the duration of the complete experiment and the effect of low temperature on the microorganisms. However, the contamination concentrations were sufficiently high to evaluate our hypothesis.

\section{Conclusions}

In conclusion, this report presents promising initial results for the use of HHP in the disinfection of bone grafts. It underlines the importance of further preclinical evaluation of its efficacy in other microorganisms and strains as well as its influence on bone remodelling and the structural integrity of bone grafts.

Acknowledgements Research presented above was supported by Bonebank, BIS-LIFE, Leiden, The Netherlands and TDI.BV Helmond, The Netherlands. Allograft femoral heads used in this experiment were provided without costs by BIS-LIFE. The costs for the microbiology experiment (2000 Euros) were covered by TDI.BV. They also provided the use of the HHP without costs. Sponsors mentioned above were consulted before the start of the experiment and were allowed to view the results and use them appropriately. There were NO Content, Structural, Textual or Publication restrictions. The funders had an advisory role in study design, but not in data collection and analysis, decision to publish, or preparation of the manuscript.

\section{Compliance with ethical standards}

Competing interests TDI.BV Helmond, The Netherlands has drafted a patent application number (P100159NL00), for HHP use in allograft disinfection. We received funding form a source: TDI.BV Helmond, The Netherlands and BIS-LIFE, Leiden, The Netherlands. This does not alter our adherence to Biomaterials policies on sharing data and materials.

Open Access This article is distributed under the terms of the Creative Commons Attribution 4.0 International License (http:// creativecommons.org/licenses/by/4.0/), which permits unrestricted use, distribution, and reproduction in any medium, provided you give appropriate credit to the original author(s) and the source, provide a link to the Creative Commons license, and indicate if changes were made.

\section{References}

Alpas H, Kalchayanand N, Bozoglu F, Sikes A, Dunne CP et al (1999) Variation in resistance to hydrostatic pressure among strains of food-borne pathogens. Appl Environ Microbiol 65(9):4248-4251

Barth HD, Zimmermann EA, Schaible E, Tang SY, Alliston T et al (2011) Characterization of the effects of X-ray irradiation on the hierarchical structure and mechanical properties of human cortical bone. Biomaterials 32(34):8892-8904

Chiu CK, Lau PY, Chan SWW, Fong CM, Sun LK (2004) Microbial contamination of femoral head allografts. Hong Kong Med J 10(6):401-405

de Souza AR, da Costa Demonte ALSSM, de Araujo Costa K, Faria MAC, Durães-Carvalho R et al (2013) Potentiation of high hydrostatic pressure inactivation of Mycobacterium by combination with physical and chemical conditions. Appl Microbiol Biotechnol 97(16):7417-7425

Deijkers RL, Bloem RM, Petit PL, Brand R, Vehmeyer SB et al (1996) Contamination of bone allografts: analysis of incidence and predisposing factors. J Bone Joint Surg $\mathrm{Br}$ 79(1):161-166

Diehl P, Schmitt M, Blümelhuber G, Frey B, Van Laak S et al (2003) Induction of tumor cell death by high hydrostatic pressure as a novel supporting technique in orthopedic surgery. Oncol Rep 10(6):1851-1855

Diehl P, Schmitt M, Schauwecker J, Eichelberg K, Gollwitzer H et al (2005) Effect of high hydrostatic pressure on biological properties of extracellular bone matrix proteins. Int $\mathbf{J}$ Mol Med 16(2):285-289

Diehl P, Naal F-D, Schauwecker J, Steinhauser E, Milz S et al (2006) Biomechanical properties of articular cartilage after high hydrostatic pressure treatment. Biomed Tech (Berl) 51(1):8-14

Diehl P, Schauwecker J, Eichelberg K, Gollwitzer H, Magdolen U et al (2007) Quantitative analysis of the impact of shorttime high hydrostatic pressure on bone tumor-associated proteases. Int J Mol Med 19(4):667-673

Diehl P, Schauwecker J, Mittelmeier W, Schmitt M (2008) High hydrostatic pressure, a novel approach in orthopedic surgical oncology to disinfect bone, tendons and cartilage. Anticancer Res 28(6B):3877-3883

Gollwitzer H, Mittelmeier W, Brendle M, Weber P, Miethke T et al (2009) High hydrostatic pressure for disinfection of bone grafts and biomaterials: an experimental study. Open Orthop J 3:1-7

Graham JM, Ayati BP, Holstein SA, Martin JA (2013) The role of osteocytes in targeted bone remodeling: a mathematical model. PLoS ONE 8(5):e63884-4

Journeaux SF, Johnson N, Bryce SL, Friedman SJ, Sommerville $\mathrm{SM}$ et al (1998) Bacterial contamination rates during bone allograft retrieval. J Arthroplasty 14(6):677-681

Kaminski A, Jastrzebska A, Grazka E, Marowska J, Gut G et al (2012) Effect of gamma irradiation on mechanical properties of human cortical bone: influence of different processing methods. Cell Tissue Bank 13(3):363-374

Kishida T, Cui F-D, Ohgitani E, Gao F, Hayakawa K et al (2013) High pressure treatment under subfreezing temperature results in drastic inactivation of enveloped and non-enveloped viruses. Biotechnol Lett 35(8):1297-1301

Knorr D (1999) Novel approaches in food-processing technology: new technologies for preserving foods and modifying function. Curr Opin Biotechnol 10(5):485-491 
Mathijssen NMC, Sturm PD, Pilot P, Bloem RM, Buma P et al (2013) Comparison of contamination of femoral heads and pre-processed bone chips during hip revision arthroplasty. Cell Tissue Bank 14(4):615-620

Mitchell EJ, Stawarz AM, Kayacan R, Rimnac CM (2004) The effect of gamma radiation sterilization on the fatigue crack propagation resistance of human cortical bone. J Bone Joint Surg Am 86-A(12):2648-2657

Naal F-D, Mengele K, Schauwecker J, Gollwitzer H, Gerdesmeyer L et al (2005) High hydrostatic pressure-induced cell death in human chondrocytes and chondrosarcoma cells. Anticancer Res 25(3B):1977-1982

Naal FD, Schauwecker J, Steinhauser E, Milz S, von Knoch F et al (2008) Biomechanical and immunohistochemical properties of meniscal cartilage after high hydrostatic pressure treatment. J Biomed Mater Res Part B Appl Biomater 87(1):19-25

Nguyen H, Morgan DAF, Sly LI, Benkovich M, Cull S et al (2008) Validation of $15 \mathrm{kGy}$ as a radiation sterilisation dose for bone allografts manufactured at the Queensland Bone Bank: application of the VDmax 15 method. Cell Tissue Bank 9(2):139-147

Nguyen H, Cassady AI, Bennett MB, Gineyts E, Wu A et al (2013) Reducing the radiation sterilization dose improves mechanical and biological quality while retaining sterility assurance levels of bone allografts. Bone 57(1):194-200

Olivier SA, Bull MK, Stone G, van Diepenbeek RJ, Kormelink F et al (2011) Strong and consistently synergistic inactivation of spores of spoilage-associated Bacillus and Geobacillus spp. by high pressure and heat compared with inactivation by heat alone. Appl Environ Microbiol 77(7):2317-2324
Pajevic PD, Powell WF, Barry KJ, Bringhurst FR (2010) Osteocytes and calcium homeostasis. Bone 47:S23-3

San Martín MF, Barbosa-Cánovas GV, Swanson BG (2002) Food processing by high hydrostatic pressure. Crit Rev Food Sci Nutr 42(6):627-645

Schauwecker J, Von Eisenhart-Rothe R, Burgkart R, Tuebel J, Schmitt M et al (2011) Revitalization of human bone after extracorporeal high hydrostatic pressure treatment. Anticancer Res 31(4):1235-1239

Schubert T, Bigaré E, Van Isacker T, Gigi J, Delloye C et al (2012) Analysis of predisposing factors for contamination of bone and tendon allografts. Cell Tissue Bank 13(3):421-429

Sommerville SM, Johnson N, Bryce SL, Journeaux SF, Morgan DA (2000) Contamination of banked femoral head allograft: incidence, bacteriology and donor follow up. Aust $\mathrm{N}$ Z J Surg 70(7):480-484

Tomford WW, Thongphasuk J, Mankin HJ, Ferraro MJ (1990) Frozen musculoskeletal allografts. A study of the clinical incidence and causes of infection associated with their use. J Bone Joint Surg Am 72(8):1137-1143

van de Pol GJ, Sturm PDJ, van Loon CJ, Verhagen C, Schreurs BW (2007) Microbiological cultures of allografts of the femoral head just before transplantation. J Bone Joint Surg Br 89(9):1225-1228

Weber P, Diehl P, Hofmann GO, Miethke T, Schmitt-Sody M et al (2008) Extracorporeal high hydrostatic pressure as a new technology for the disinfection of infected bone specimens. Biomed Tech (Berl) 53(4):190-198 